\title{
Combined epigenetic/genetic study identified an ALS age of onset modifier
}

\author{
Ming Zhang ${ }^{1,2,3,4^{*}}$, Zhengrui $\mathrm{Xi}^{2}$, Sara Saez-Atienzar ${ }^{5}$, Ruth $\mathrm{Chia}^{5}$, Danielle Moreno ${ }^{2}$, Christine Sato ${ }^{2}$,
} Mahdi Montazer Haghighi ${ }^{2}$, Bryan J. Traynor ${ }^{5}$, Lorne Zinman ${ }^{6,7}$ and Ekaterina Rogaeva ${ }^{2,7^{*}}$

\begin{abstract}
Age at onset of amyotrophic lateral sclerosis (ALS) is highly variable (eg, 27-74 years in carriers of the $\mathrm{G}_{4} \mathrm{C}_{2}$-expansion in C9orf72). It might be influenced by environmental and genetic factors via the modulation of DNA methylation (DNAm) at CpG-sites. Hence, we combined an epigenetic and genetic approach to test the hypothesis that some common single nucleotide polymorphisms (SNPS) at CpG-sites (CpG-SNPs) could modify ALS age of onset. Our genome-wide DNAm analysis suggested three CpG-SNPs whose DNAm levels are significantly associated with age of onset in 249 ALS patients ( $q<0.05)$. Next, genetic analysis validated the association of rs 4970944 with age of onset in the discovery $(n=469 ; P=0.025)$ and replication $(n=4160 ; P=0.007)$ ALS cohorts. A meta-analysis of the cohorts combined showed that the median onset in AA-carriers is two years later than in GG-carriers $(n=4629 ; P=0.0012)$. A similar association was observed with its tagging SNPs, implicating a $16 \mathrm{~Kb}$ region at the $1 \mathrm{q} 21.3$ locus as a modifier of ALS age of onset. Notably, rs4970944 genotypes are also associated with age of onset in C9orf72-carriers $(n=333 ; P=0.025)$, suggesting that each A-allele delays onset by 1.6 years. Analysis of Genotype-Tissue Expression data revealed that the protective A-allele is linked with the reduced expression of CTSS in cerebellum ( $P=0.00018)$, which is a critical brain region in the distributed neural circuits subserving motor control. CTSS encodes cathepsin $\mathrm{S}$ protein playing a key role in antigen presentation. In conclusion, we identified a $16 \mathrm{~Kb}$ locus tagged by rs4970944 as a modifier of ALS age of onset. Our findings support the role of antigen presenting processes in modulating age of onset of ALS and suggest potential drug targets (eg, CTSS). Future replication studies are encouraged to validate the link between the locus tagged by rs4970944 and age of onset in independent ALS cohorts, including different ethnic groups.
\end{abstract}

Keywords: ALS, Age of onset, Modifier, DNA methylation, CpG-SNPs, Genetic association

\section{Introduction}

Amyotrophic lateral sclerosis (ALS) is characterized by the progressive degeneration of upper and lower motor neurons in the brain and spinal cord, leading to paralysis [6]. About $90 \%$ of patients have sporadic ALS. Genetic

\footnotetext{
*Correspondence: mingzhang@tongji.edu.cn; ekaterina.rogaeva@utoronto. ca

1 Shanghai First Rehabilitation Hospital, School of Medicine, Tongji University, Shanghai 200090, China

${ }^{2}$ Tanz Centre for Research in Neurodegenerative Diseases, University of Toronto, 60 Leonard Ave., Toronto, ON M5T 0S8, Canada

Full list of author information is available at the end of the article
}

mutations explain 10-20\% sporadic and $\sim 50 \%$ familial ALS, mainly caused by the most common ALS genes (C9orf72, SOD1, TARDBP and FUS) [6, 30]. Patients with ALS have variable clinical presentation, including disease duration and age or site of onset [30]. For example, carriers of the $\mathrm{G}_{4} \mathrm{C}_{2}$-expansion in $\mathrm{C} 9$ orf 72 have been reported to have disease onset between 27 and 74 years and duration of $0.5-22$ years [10].

Disease phenotype can be modified by DNA methylation (DNAm) at CpG dinucleotides, which is one of the key epigenetic modifications regulating gene expression or RNA splicing. For instance, smoking and head original author(s) and the source, provide a link to the Creative Commons licence, and indicate if changes were made. The images or other third party material in this article are included in the article's Creative Commons licence, unless indicated otherwise in a credit line to the material. If material is not included in the article's Creative Commons licence and your intended use is not permitted by statutory regulation or exceeds the permitted use, you will need to obtain permission directly from the copyright holder. To view a copy of this licence, visit http://creativecommons.org/licenses/by/4.0/. The Creative Commons Public Domain Dedication waiver (http://creativeco mmons.org/publicdomain/zero/1.0/) applies to the data made available in this article, unless otherwise stated in a credit line to the data. 
injury (suggested ALS risk factors $[8,31]$ ) are linked to DNAm [20]. Furthermore, DNAm is closely associated with aging-the strongest risk factor of ALS [3]. Specifically, the cumulative assessment of DNAm levels at 353 age-related CpGs (constituting DNAm-age) revealed an association of DNAm-age acceleration with disease age of onset, duration or survival in C9orf72-carriers and general ALS patients [35, 36]. DNAm-age is not greatly modulated by genetic variations, because none of the 353 age-related CpGs are mapped to common single nucleotide polymorphisms (SNPs) with a minor allele frequency $(\mathrm{MAF})>5 \%$ [3] .

In general, DNAm levels at certain GpGs could be modified by genetic factors. The strong genetic control of DNAm is evident by the very similar methylome pattern in identical twins/triplets vs fraternal siblings [33, 37]. A specific example is increased DNAm at the C9orf72 locus in response to a $\mathrm{G}_{4} \mathrm{C}_{2}$-expansion, which correlates with disease duration and age of onset [14, 27, 32]. However, it is largely unknown if other genetic variants that alter DNAm are linked to ALS phenotypes.

Importantly, CpG-sites are mutational hotspots, because methyl-C can spontaneously deaminate to $\mathrm{T}$ [19]. SNPs causing the gain/loss of CpG-sites (CpGSNPs) are linked to DNAm level and could modify disease phenotype [34]. CpG-SNPs contribute largely to allele-specific methylation, which is linked to gene expression, transcription factor binding, and associated with some mental illnesses (eg, schizophrenia) [13, 15]. Our prior study of CpG-SNPs revealed the C6orf10 locus as an age of onset modifier in C9orf72-carriers, but not in C9orf72 negative ALS patients [34]. SNPs in C6orf10 are linked with the frontal cortex expression of $H L A-D R B 1$ (a proinflammation and antigen-presenting gene) [34]. It is unknown if expression quantitative trait loci (eQTL) of other antigen-presenting genes are linked with ALS age of onset, which was in part addressed in the current study.

Here, we used an integrated epigenetic and genetic approach to identify functional genetic variants associated with age of onset in ALS patients.

\section{Materials and methods ALS participants}

Cohort characteristics of unrelated ALS patients are presented in Table 1. The discovery cohort included 469 Canadian ALS patients (without causal mutations in C9orf72, SOD1, TARDBP or FUS). The replication cohort consisted of 4160 US ALS patients, including 333 C9orf72-carriers. All patients are of Caucasian origin and diagnosed with ALS using the El Escorial revisited clinical criteria [5]. ALS age of onset was defined as the
Table 1 Sample characteristics for unrelated ALS patients included in the DNA methylation (DNAm) study, and genotyping analysis of candidate variants in discovery and replication stage

\begin{tabular}{|c|c|c|c|}
\hline Sample characteristics & $\begin{array}{l}\text { DNAm } \\
\text { analysis } \\
(n=249)\end{array}$ & $\begin{array}{l}\text { Genotyping } \\
\text { analysis } \\
(n=469)\end{array}$ & $\begin{array}{l}\text { Replication } \\
\text { cohort } \\
(n=4160)\end{array}$ \\
\hline Familial ALS (n, \%) & $49,19.6 \%$ & $68,14.5 \%$ & $415,10.0 \%$ \\
\hline Sporadic ALS (n, \%) & $200,80.3 \%$ & $401,85.5 \%$ & $3745,90 \%$ \\
\hline Sex, male $(n, \%)$ & $148,59.4 \%$ & $280,59.7 \%$ & $2517,60.5 \%$ \\
\hline $\begin{array}{l}\text { Median age of onset } \\
\text { (interquartile range), } \\
\text { years }\end{array}$ & $60(51-69)$ & $61(52-68)$ & $58(49-66)$ \\
\hline Bulbar site of onset (n) & 67 & 112 & 990 \\
\hline Limb site of onset (n) & 172 & 314 & 2882 \\
\hline
\end{tabular}

self-reported age at which the first limb (spinal) or bulbar symptom appeared.

\section{Procedures}

We analyzed genome-wide DNAm data of $\sim 850,000$ DNAm-sites from the EPIC BeadChip (Illumina) previously generated using bisulfite converted blood DNA of Canadian ALS patients [35]. We used the minfi package in R-project [2] to pre-process the raw data and select common CpG-SNPs (MAF $>5 \%$ ). The $\beta$-value was used to estimate the DNAm level of each CpG-site. We included common CpG-SNPs with a difference between maximum and minimum $\beta$-value $>0.5$ (considering the effect of SNPs on DNAm) and used the gaphunter function (minfi package) [1] to study CpGs with a multimodal distribution of DNAm level.

For the genetic analysis, we used blood DNA. In the discovery cohort, genotypes for candidate SNPs were obtained by multiplex genotyping using iPlex (Agena Bioscience) and MassArray Analyzer 4 at the Clinical Genomics Centre (Toronto, Canada). For the replication cohort, genome-wide genotyping was performed in the Laboratory of Neurogenetics, National Institutes of Health using HumanOmniExpress (version 1.0 genotyping 716,503 SNPs) according to the manufacturer's protocol (Illumina Inc., San Diego, CA). The 34,335 US controls (71\% females; mean age 65 with a standard deviation of 13 years) were previously genotyped on HumanOmni BeadChips (Illumina) [23]. In the replication stage, 4 SNPs (Table 2) were either genotyped or imputed using the Michigan Imputation Server pipeline employing Minimac4 [12] based on the Haplotype Reference Consortium r1.1 2016 [22] (http://www.haplotypereference-consortium.org).

To measure the degree of linkage disequilibrium (LD), we extracted $R^{2}$ values (range from 0 to 1 , indicating the highest LD) from the LDlink web tool by selecting 
Table 2 The association of rs4970944 and its tagging variants with ALS age of onset in the discovery and replication cohorts

\begin{tabular}{|c|c|c|c|c|c|c|c|c|}
\hline \multirow[t]{2}{*}{ SNP } & \multirow[t]{2}{*}{ Location (hg19) } & \multirow[t]{2}{*}{ MAF } & \multicolumn{3}{|c|}{ Discovery stage $(n=469)$} & \multicolumn{3}{|c|}{ Replication stage $(n=4160)$} \\
\hline & & & B & SE & $P$ value & B & SE & $P$ value \\
\hline rs4970944 & chr1:151,163,317 & 0.32 & 2 & 0.9 & 0.025 & 0.8 & 0.3 & 0.007 \\
\hline rs10888406 & chr1:151,166,896 & 0.32 & 2 & 0.9 & 0.025 & 0.8 & 0.3 & 0.007 \\
\hline rs11204785 & chr1:151,150,857 & 0.33 & NA & NA & NA & 0.7 & 0.3 & 0.013 \\
\hline rs11807075 & chr1:151,153,806 & 0.33 & NA & NA & NA & 0.7 & 0.3 & 0.014 \\
\hline
\end{tabular}

Results from the additive multivariate linear regression model are presented (adjusted for sex, ALS site of onset and family history). Non-Finnish European minor allele frequencies (MAF) were extracted from the gnomAD database $(N A=$ not available, $B=$ linear regression coefficient $B e t a, S E=$ standard error)

the 1000 Genomes European population data (https:// ldlink.nci.nih.gov/?tab=home). We searched for known variants within the boundaries of the LD-block $\left(R^{2}>0.9\right)$ tagged by the top significant SNP (rs4970944) using the 'proxy search. The LD-block was also analyzed for transcription factor binding sites and DNase I hypersensitivity using the UCSC genome browser. We searched for eQTL using Genotype-Tissue Expression data (GTEx v8) from 49 types of human tissue [9]. We used the GTEx portal (https://www.gtexportal.org/) to analyze the association between rs4970944 genotypes and gene expression in specific tissues by a linear regression method. Normalized effect size (NES) was defined as the slope of the linear regression.

To understand which cell types are linked to CTSS gene expression in brain tissue, we analyzed publicly available single nuclei RNA sequencing data of 8 human entorhinal cortex samples (GEO: GSE138852). The Seurat package [7] in R was used to perform quality control, pre-processing, normalization, and dimensional reduction/clustering by the Uniform Manifold Approximation and Projection (UMAP) technique.

\section{Statistics}

We used the linear regression model in $\mathrm{R}$ to assess the genome-wide association between DNAm levels of CpGSNPs and ALS age of onset, and calculated the false discovery rate to obtain adjusted q-values [34]. Adjustments for sex, site of onset, DNAm-age acceleration and the top 5 principal components (PCs) were also performed. The PCs were generated by PC analysis of common CpGSNPs with the prcomp function in R. The Manhattan plot was used to prioritize significant CpG-SNPs $(\mathrm{q}<0.05)$ for further genetic study. A QQ plot was generated and the genomic inflation factor was estimated, using the $\mathrm{R}$ qqman package [11].

To analyze the association between genotypes and age of onset, we used an additive multivariate linear regression model adjusting for sex, site of onset, family history, and/or DNAm-age acceleration. We presented the linear regression coefficient Beta (B) with standard error (SE). We used the Mann Whitney $U$ test to evaluate differences in age of onset in the ALS subgroups (Table 1) stratified by sex (males vs females), site of onset (limb vs bulbar onset), ALS family history (familial vs sporadic) or C9orf72 status (expansion vs wild-type). We also conducted a metaanalysis (R metafor package) [34] with a fixed-effect model to assess the pooled effect size of the coefficient $B$ for the discovery and replication stages. $\mathrm{R}$ project 4.0 .0 was used for statistical analysis. Results with a $P$-value $<0.05$ were accepted as statistically significant.

\section{Results \\ Discovery stage suggested candidate CpG-SNP linked to ALS age of onset}

The study design is presented in Fig. 1. First, we conducted a genome-wide DNAm analysis of CpG-SNPs in 249 Canadian ALS patients (Table 1). Among the 4300 common CpG-SNPs with a multimodal distribution, the DNAm levels at $10 \mathrm{CpG}$-SNPs were associated with age of onset at a false discovery rate $<0.05$, three of which remained significant after adjustment for sex, site of onset, DNAm-age acceleration and the top 5 PCs (Fig. 2a, b, Additional file 1: Table S1, Fig. S1).

Next, we conducted a genetic analysis of candidate variants detected during the DNAm study in an expanded Canadian ALS cohort $(\mathrm{n}=469$; Table 1$)$. Only rs4970944 and its tagging SNP (rs10888406) showed significant association with ALS age of onset (Table 2). Each A-allele of rs4970944 is linked to a 2-year later onset (adjusted $\mathrm{P}=0.025, \mathrm{~B}=2.0, \mathrm{SE}=0.9$ ) (Fig. 3a, Table 2). In the original subgroup with DNAm data $(n=249)$, multivariate linear regression analysis confirmed that the genotypes of rs4970944 are associated with both DNAm level and age of onset (Fig. 2c). Specifically, rs4970944 genotypes control the gain or loss of DNAm at CpG-site cg15625495 $\left(\mathrm{P}=2 \times 10^{-16}\right)$, thereby underlying the association with age of onset (adjusted $\mathrm{P}=0.003, \mathrm{~B}=3.0, \mathrm{SE}=1.0$ ). 


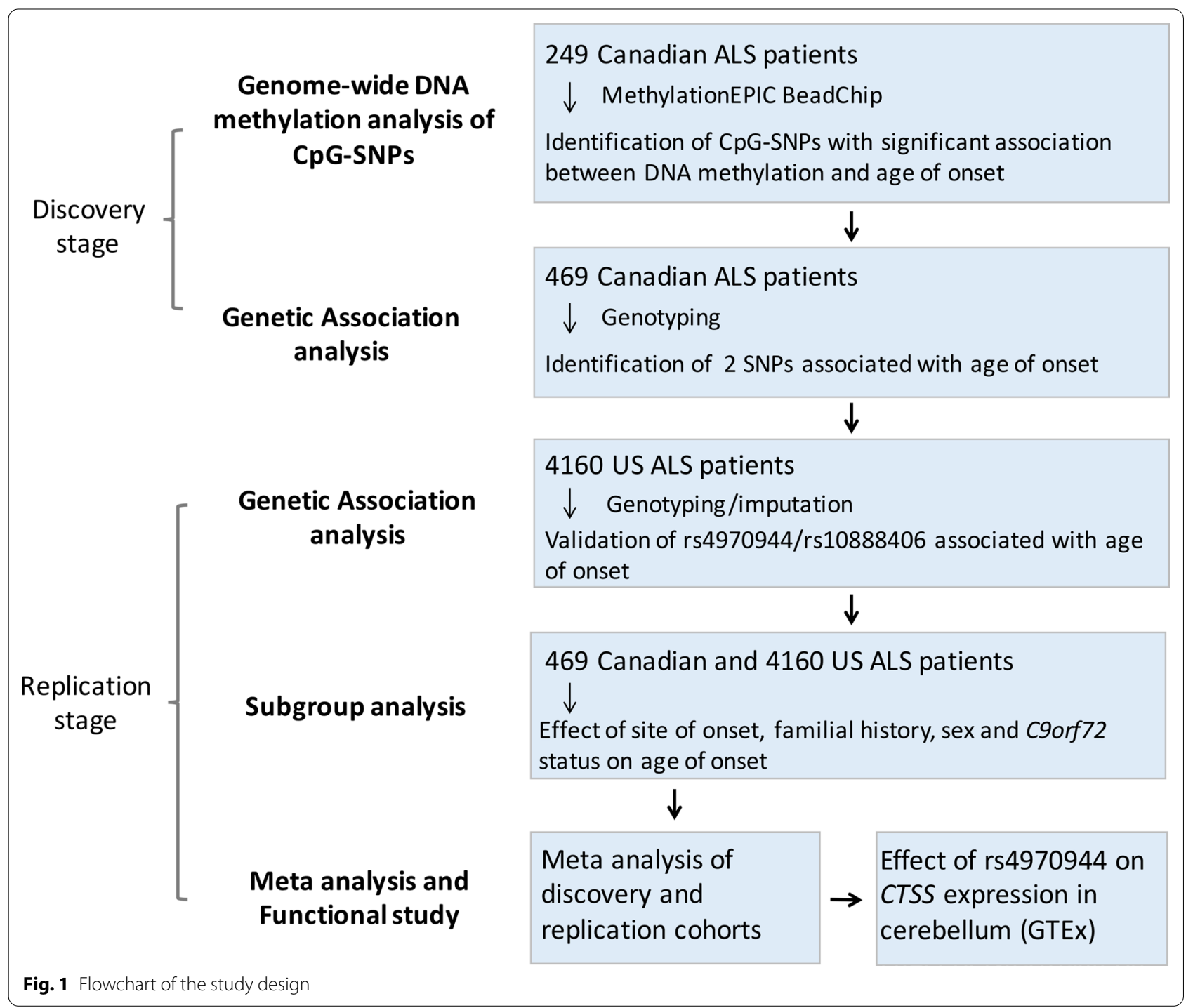

\section{Replication stage validated the link of ALS age of onset with LD-block tagged by rs4970944}

In a US cohort of 4160 ALS patients (Table 1), we validated the association with age of onset for rs4970944 after adjustment for sex, site of onset, and family history $(\mathrm{P}=0.007, \mathrm{~B}=0.8, \mathrm{SE}=0.3)$, which suggested that each A-allele of rs4970944 delays ALS onset by 0.8 year (Table 2, Fig. 3b). Four SNPs in strong LD with rs4970944 $\left(\mathrm{R}^{2}>0.9\right)$ implicate a $16 \mathrm{~Kb}$ LD-block (chr1:151,150,857-151,166,896) partially overlapping VPS72 (Additional file 1: Fig. S2). Genotypes for three of them (rs10888406, rs11204785, rs11807075) were available in the replication dataset and associated with age of onset (adjusted $\mathrm{P}=0.007-0.014 ; \mathrm{B}=0.7-0.8$, $\mathrm{SE}=0.3$ ) (Table 2, Additional file 1: Fig. S3). Notably, the $16 \mathrm{~Kb}$ LD-block tagged by rs4970944 is associated with ALS age of onset, but not with ALS risk in our case-control study (4160 US ALS patients and 34,335 controls; $\mathrm{P}=0.93)$.

\section{Subgroup analyses and the association of rs4970944 with ALS age of onset in C9orf72-carriers}

We performed a subgroup analysis for sex, family history and site of onset in the discovery and replication cohorts. We observed a significantly younger onset in ALS patients with limb vs bulbar onset $(P=0.0016$ for Canadian patients; $\mathrm{P}=2.2 \times 10^{-16}$ for US patients) or with familial vs sporadic ALS ( $\mathrm{P}=0.0005$ for Canadian patients; $\mathrm{P}=3.1 \times 10^{-13}$ for US patients) (Additional file 1: Fig. S4-S5, Table S2). Males showed a significantly 

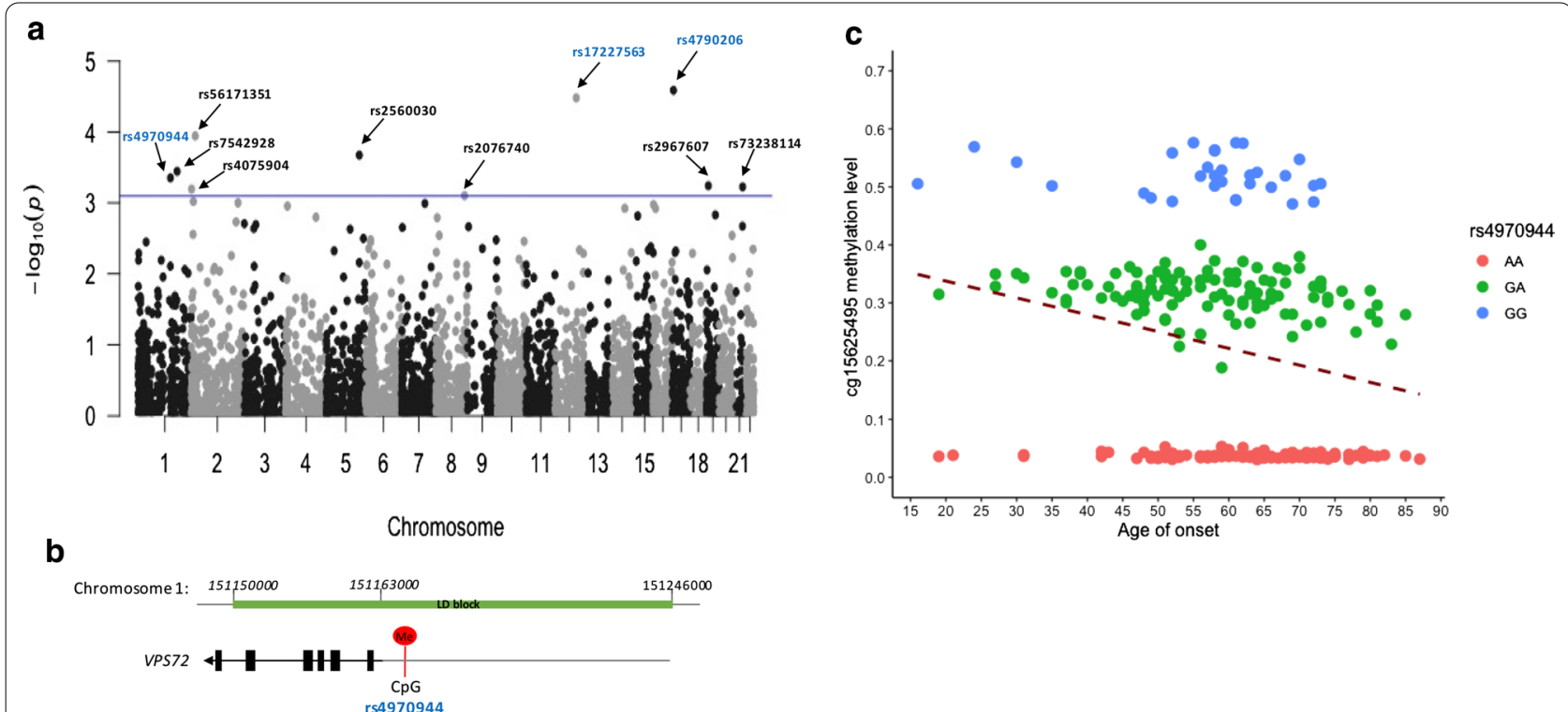

Fig. 2 Genome-wide DNA methylation (DNAm) analysis and genetic association study suggested candidate CpG-SNPs associated with ALS age of onset. a Manhattan plot presenting the association between DNAm levels of common CpG-SNPs and ALS age of onset. Three CpG-SNPs in blue remained significant after adjusting for sex, site of onset, DNAm-age acceleration and the top 5 principal components. The blue line represents the trend of statistical significance (false discovery rate $<0.05$ ). b The locus on chr1:151,150,000-151,246,000 tagged by rs4970944 associated with ALS age of onset. Arrows indicate the transcriptional direction of VPS72 (5' to $\left.3^{\prime}\right)$. "Me" in red represents the CpG-site, methylation of which is affected by rs4970944. The linkage disequilibrium (LD) block tagged by rs4970944 ( $\left.R^{2}>0.8\right)$ is highlighted in green. c Genotypes of rs4970944 are significantly associated with DNAm status: $P=2 \times 10^{-16}, B=-0.25$ (SE: 0.003 ); and age of onset: $P=0.003$ adjusted for sex, family history, site of onset and DNAm-age acceleration, $B=3.0$ (SE: 1.0). The dashed line represents the linear regression trend

younger onset than females in the US cohort $(\mathrm{P}=0.001$, Additional file 1: Fig. S5, Table S2), but not in the more modest Canadian cohort $(\mathrm{P}=0.7$, Additional file 1: Fig. S4, Table S2). Notably, a subgroup analysis for the 4030 cases with known status for the expansion in C9orf72 did not detect a significant difference in age of onset between C9orf72-carriers $(\mathrm{n}=333)$ vs non-carriers (Additional file 1: Fig. S6a).

After adjustment for sex, site of onset and ALS family history, rs4970944 is significantly associated with age of onset in both C9orf72-carriers $(\mathrm{P}=0.025, \mathrm{~B}=1.6$, $\mathrm{SE}=0.7 ; \mathrm{n}=333)$ and non-carriers $(\mathrm{P}=0.015 ; \mathrm{B}=0.8$, $\mathrm{SE}=0.3 ; \mathrm{n}=3697$ ) (Additional file 1: Fig. S6b-6c). It suggested that each A-allele of rs4970944 delays ALS onset by 1.6 years in C9orf72-carriers. The median onset in AA-carriers is 2.5 years later than GG-carriers: 57.5 years with an interquartile range (IQR) of 52-64 vs 55.0 years (IQR: 50-59). The genotypes of tagging SNPs (rs10888406, rs11807075, rs11204785) showed a similar association with ALS onset in C9orf72-carriers $(\mathrm{P}=0.018-0.025, \mathrm{~B}=1.6-1.7, \mathrm{SE}=0.7)$.

\section{Meta/pooled-analyses revealed overall effect of rs 4970944 tagging SNPs on ALS age of onset}

To estimate the overall effect size of rs4970944 on age of onset, we conducted a meta-analysis of the adjusted coefficient Beta (B) in the 4629 ALS patients from the discovery and replication stages using a fixed-effects model. We observed that every A-allele of rs4970944 is linked to 0.9-year later onset (pooled $\mathrm{B}=0.9, \mathrm{P}=0.0012$ ) (Fig. 3c), and the same result was obtained in the 4166 C9orf72 negative patients (pooled $\mathrm{B}=0.9, \mathrm{P}=0.0015$, Additional file 1: Fig. S8). A pooled linear regression analysis suggested a similar effect of the A-allele on age of onset (adjusted $\mathrm{P}=0.001, \mathrm{~B}=0.9, \mathrm{SE}=0.3$ ) (Additional file 1: Fig. S7). The median onset in AA-carriers was 2 years later than GG-carriers: 59 years (IQR:50-76) vs 57 years (IQR:49-65). The same result was observed for tagging SNP rs10888406 (Additional file 1: Fig. S3).

\section{Genotypes of rs4970944 are associated with microglia related CTSS expression in cerebellum}

We conducted a bioinformatics analysis of the top SNPs tagged by rs4970944 $\left(R^{2}>0.9\right)$ and found that none of them overlap transcription factor binding sites or DNase I hypersensitivity sites (Additional file 1: Table S3). To explore if rs4970944 genotypes could modify ALS age of onset by regulating gene expression, we analyzed the GTEx eQTL dataset [9], consisting of 49 tissues from up to 670 individuals. We did not observe an association of rs4970944 with expression of VPS72 (the gene partially overlapping the LD-block tagged by rs4970944), which 


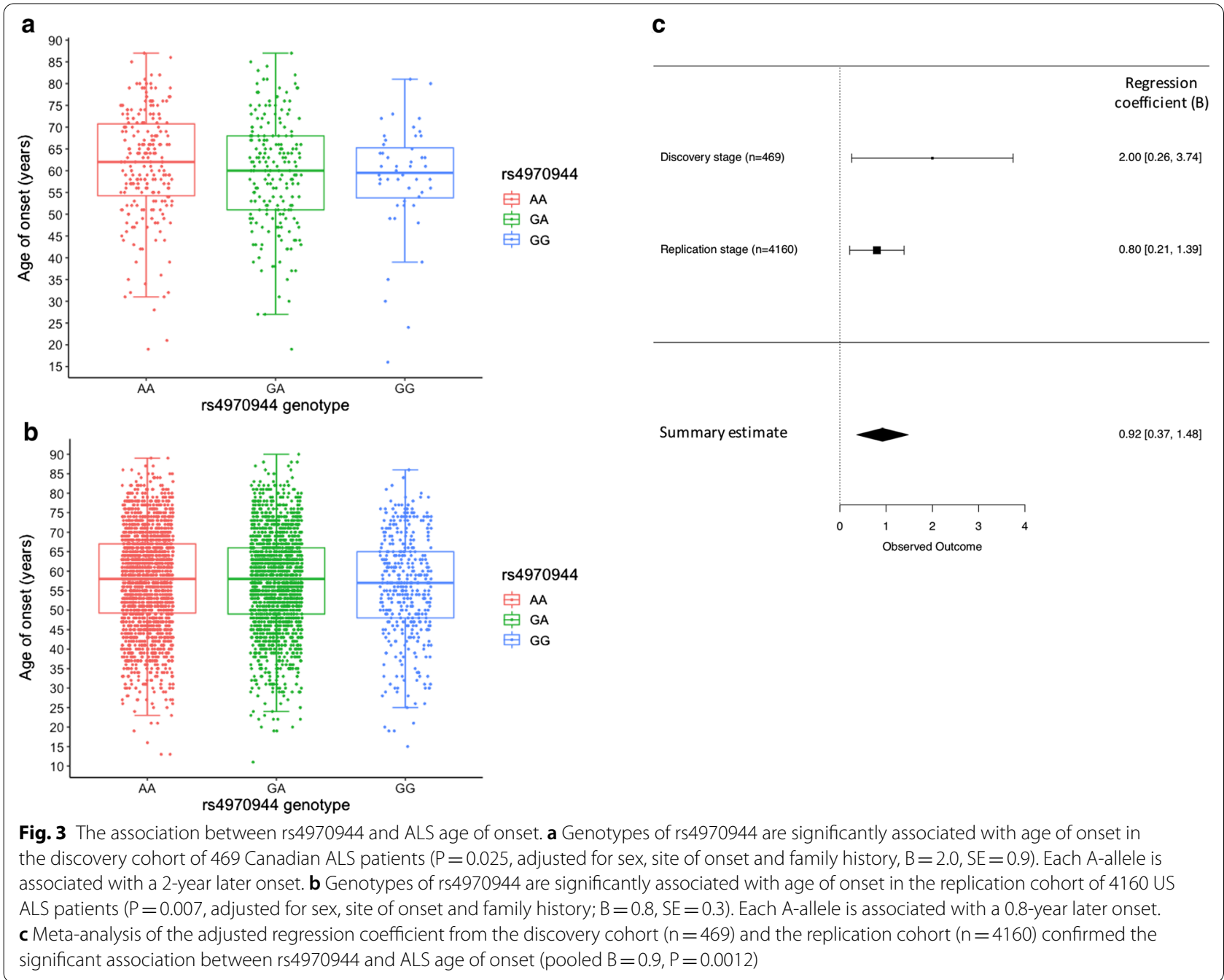

is not surprising since the closest gene to the significant variant is often not the functional disease-gene [18, 34]. However, rs4970944 genotypes are associated with the expression of adjacent genes in a wide range of tissues (Additional file 1: Table S4). Importantly, the A-allele of rs4970944 (linked to a later ALS onset) is significantly associated with reduced expression of CTSS in cerebellum ( $\mathrm{P}=0.00018, \mathrm{NES}=-0.31, \mathrm{n}=209$; Additional file 1: Fig. S9), with similar results for its tagging SNPs $(\mathrm{P}=0.00018, \mathrm{NES}=-0.31$ for $\mathrm{rs} 10888406 ; \mathrm{P}=0.000056$, $\mathrm{NES}=-0.34$ for $\mathrm{rs} 11204785 ; \mathrm{P}=0.000059, \mathrm{NES}=-0.33$ for rs11807075). For other ALS-related tissues available in the GTEx dataset (eg, spinal cord), we did not observe a significant association with rs4970944.

Single nuclei RNA sequencing analysis suggested that CTSS expression is enriched in a gene cluster expressing CD74, DOCK8, C10orf11, ST6GAL1, and ARHGAP24, most of which were reported to be expressed in microglia experimentally (Additional file 1: Fig. S10). We also used a UMAP to visualize the gene expression of CTSS and selected genes, including ALS genes (C9orf72, SOD1), microglial genes (TREM2, CD33) and the antigen-presenting gene (HLA-DRB1). It showed that microglial/ antigen-presenting genes (TREM2, CD33, HLA-DRB1) and CTSS are enriched in the same cluster (Additional file 1: Fig. S11).

\section{Discussion}

Allele-specific methylation (eg, at CpG-SNPs) could help annotate the functional effects of non-coding variants and prioritize candidates as disease risk variants [15]. Hence, we searched for CpG-SNPs associated with ALS age of onset using an innovative strategy combining DNAm and genetic data [34], which could reveal even modest associations often overlooked in genome-wide association studies due to excessive correction for multiple testing leading to the loss of true positive signals. In our large ALS cohort $(n=4629)$, we detected a modest 
but significant effect on age of onset of SNPs at an LDblock tagged by rs4970944. The median onset in AA-carriers of rs4970944 was 2 years later than GG-carriers (59 vs 57 years). This association has even a stronger effect size $(\mathrm{B}=1.6)$ in 333 C9orf72-carriers, indicating that a larger dataset is needed to search for phenotype modifiers in heterogeneous sporadic patients compared to carriers of the same mutation.

The GTEx database can suggest the link(s) between genetic variations and gene expression levels across a diverse set of human tissues, providing a powerful approach for analyzing eQTLs and inferring the downstream effects of phenotype associated variants [9]. Using the GTEx database, the current study revealed that rs4970944 and its tagging SNPs are eQTLs. In contrast to the protective A-allele, the G-allele of rs4970944 (associated with an earlier ALS onset) is linked to higher CTSS expression in cerebellum, which is a critical region in the distributed neural circuits subserving motor control and cognitive processing [28]. Notably, brain expression of C9orf72 is greatest in cerebellum [25], and C9orf72-carriers show a high burden of dipeptide repeat inclusions in cerebellum [21], suggesting that it is an important brain region linking C9orf72 pathology and the CTSSassociated adaptive immune response. However, the connection between rs4970944 and gene expression in other disease-relevant tissues remains to be comprehensively explored. Notably, elevated CTSS expression was reported in the anterior lumbar spinal cord of ALS patients [4] and brain tissue of patients with Alzheimer's Disease [24].

CTSS encodes Cathepsin S protein, which is a cysteine endoprotease removing the invariant chain from major histocompatibility complex class II molecules, regulating antigen presentation and immunity [26]. Our single nuclei RNA sequencing analysis and a previous report [29] support the notion that CTSS might be a microglia specific gene. Of note, higher expression of another microglia-expressing antigen-presenting gene ( $H L A$ DRB1) was also linked to an earlier onset in C9orf72 patients [34]. Together, it suggests the important role of immune system genes in ALS and other neurodegenerative diseases. For example, several genes causing ALS and/or frontotemporal dementia (C9orf72, VCP, SQSTM1, OPTN, UBQLN2, GRN, CHMP2B) affect the autophagic machinery [16]; and some Alzheimer's Disease genes (APOE, TREM2, CD33, ABCA7) are preferentially or exclusively expressed in microglia [17]. Hence, more studies are needed to understand the role of both the innate and adaptive immune pathways in neurodegenerative diseases.

Ongoing international MinE projects (https://www. projectmine.com) aim to characterize both genome and methylome data of ALS cases, which might be used to validate our findings in different ethnic groups. Moreover, it remains to be investigated if the rs4970944-locus plays a role in ALS-related diseases, such as frontotemporal dementia. It would require a large dataset, since the age at onset of frontotemporal dementia is obtained from unaffected family members, in contrast to the more accurate self-reported onset in ALS.

\section{Conclusions}

We identified a $16 \mathrm{~Kb}$ LD-block tagged by rs4970944 as a modifier of ALS age of onset. Genotypes of rs4970944 are associated with the DNAm level at the corresponding CpG and linked to cerebellar expression of CTSS, highlighting the role of antigen presenting processes in modifying ALS onset. Our findings contribute to understanding the functional consequence of non-coding variants and suggest antigen-presenting processes (eg, involving CTSS) as potential drug targets to delay ALS onset. Since inflammation in ALS is a complex phenomenon, future studies may investigate eQTL or splicing QTL data to clarify if other genetic variants may modify disease phenotypes. Independent replication studies are also encouraged to clarify the link between ALS age of onset and the rs4970944-locus.

\section{Abbreviations}

ALS: Amyotrophic lateral sclerosis; SNPs: Single nucleotide polymorphisms; DNAm: DNA methylation; MAF: Minor allele frequency; eQTL: Expression quantitative trait loci; LD: Linkage disequilibrium; NES: Normalized effect size; PCs: Principal components; GTEx: Genotype-tissue expression project; UMAP: Uniform manifold approximation and projection; IQR: Interquartile range; B: Linear regression coefficient Beta; SE: Standard error.

\section{Supplementary Information}

The online version contains supplementary material available at https://doi. org/10.1186/s40478-021-01183-w.

Additional file 1:Table S1. Candidate CpG-SNPs with significant association between their DNAm level and age of onset in 249 ALS patients. Table S2. Results of the subgroup analysis in Canadian ALS patients $(n=469)$ and US ALS patients $(n=4160)$. Table S3. Bioinformatic annotation of SNPs in strong LD with rs4970944 $\left(R^{2}>0.9\right)$. Table S4. eQTL analysis using the GTEx database revealed significant changes in gene expression associated with rs4970944 in different tissues (normalized effect size (NES) are listed). Fig. S1. QQ plot of the genome-wide DNAm study. Fig. S2. The 16 Kb LD-block tagged by rs4970944 (chr1:151150857-151166896), including 4 SNPs (rs11204785, rs11807075, rs11299974, rs10888406) in strong LD with rs4970944 ( $\left.R^{2}>0.9\right)$. Fig. S3. Rs10888406 genotypes are significantly associated with age of onset in ALS patients in the discovery, replication and pooled sample set. Fig. S4. Subgroup analysis in Canadian ALS patients stratified for site of onset, sex and familial history. Fig. S5. Subgroup analysis in US ALS patients stratified for site of onset, sex and familial history. Fig. S6. Subgroup analysis in US ALS patients stratified for C9orf72 status. Fig. S7. Pooled analysis of the association between rs4970944 genotypes and ALS age of onset. Fig. S8. Meta-analysis of the adjusted regression coefficient from the discovery cohort $(n=469)$ and the replication cohort $(n=3697)$ in C9orf72 negative ALS patients. Fig. S9. 
Rs4970944 genotypes are significantly associated with CTSS expression in cerebellum in the GTEx database. Fig. S10. The dimension reduction figure (UMAP) of human entorhinal cortex samples. Fig. S11. Visualization of CTSS and selected genes in the dimension reduction figure (UMAP). Supplementary acknowledgements. Acknowledgements for using the dbGap dataset.

\section{Acknowledgements}

We would like to thank the patients who participated in this study. Acknowledgements for using the dbGap dataset can be found in Additional file 1: supplementary acknowledgements.

\section{Authors' contributions}

$M Z$, ER: conception and design of the work. MZ, DM, CS, MMH: conducted the genetic/epigenetic experiments. MZ, ZRX, SSA, RC: data analysis and interpretation. MZ, ER: drafting the manuscript. BJT, LZ: collection of samples and clinical information. All authors: revision of the manuscript. All authors read and approved the final manuscript.

\section{Funding}

This work was supported by the Canadian Consortium on Neurodegeneration in Aging (ER), ALS Canada (ER, LZ, MZ), the Shanghai Pujiang Program 19PJ1410300 (MZ), the National Natural Science Foundation of China (82071430) (MZ, ER), the Fundamental Research Funds for the Central Universities (MZ), and the Intramural Research Program of the $\mathrm{NIH}$, National Institute on Aging (Z01-AG000949-02) (BJT).

\section{Availability of data and material}

The datasets generated and/or analysed during the current study are available from the corresponding authors on request.

\section{Declarations}

\section{Ethics approval and consent to participate}

Informed consent was obtained from each study participant in accordance with the ethics review boards at Sunnybrook Health Sciences Centre (Canada) or the National Institute on Aging (US).

\section{Consent for publication}

Not applicable.

\section{Competing interests}

The authors report no competing interests.

\section{Author details}

${ }^{1}$ Shanghai First Rehabilitation Hospital, School of Medicine, Tongji University, Shanghai 200090, China. ${ }^{2}$ Tanz Centre for Research in Neurodegenerative Diseases, University of Toronto, 60 Leonard Ave., Toronto, ON M5T 0S8, Canada. ${ }^{3}$ Clinical Center for Brain and Spinal Cord Research, Tongji University, Shanghai 200092, China. ${ }^{4}$ Institute for Advanced Study, Tongji University, Shanghai, China. ${ }^{5}$ Neuromuscular Diseases Research Section, Laboratory of Neurogenetics, National Institute on Aging, National Institutes of Health, Bethesda, MD 20892, USA. ${ }^{6}$ Sunnybrook Health Sciences Centre, 2075 Bayview Ave, Toronto, ON M4N 3M5, Canada. ${ }^{7}$ Division of Neurology, Department of Medicine, University of Toronto, Toronto, Canada.

Received: 10 March 2021 Accepted: 14 April 2021 Published online: 23 April 2021

\section{References}

1. Andrews SV, Ladd-Acosta C, Feinberg AP, Hansen KD, Fallin MD (2016) "Gap hunting" to characterize clustered probe signals in Illumina methylation array data. Epigenet Chromatin 9:56. https://doi.org/10.1186/ s13072-016-0107-z

2. Aryee MJ, Jaffe AE, Corrada-Bravo H, Ladd-Acosta C, Feinberg AP, Hansen KD, Irizarry RA (2014) Minfi: a flexible and comprehensive Bioconductor package for the analysis of Infinium DNA methylation microarrays. Bioinformatics 30:1363-1369. https://doi.org/10.1093/bioinformatics/btu049

3. Bergsma T, Rogaeva E (2020) DNA Methylation clocks and their predictive capacity for aging phenotypes and healthspan. Neurosci Insights 15:2633105520942221. https://doi.org/10.1177/2633105520942221

4. Berjaoui S, Povedano M, Garcia-Esparcia P, Carmona M, Aso E, Ferrer I (2015) Complex Inflammation mRNA-Related Response in ALS Is Region Dependent. Neural Plast 2015:573784. https://doi.org/10.1155/2015/ 573784

5. Brooks BR, Miller RG, Swash M, Munsat TL, World Federation of Neurology Research Group on Motor Neuron D (2000) El Escorial revisited: revised criteria for the diagnosis of amyotrophic lateral sclerosis. Amyotroph Lateral Scler Other Motor Neuron Disord 1:293-299. https://doi.org/10. 1080/146608200300079536

6. Brown RH, Al-Chalabi A (2017) Amyotrophic Lateral Sclerosis. N Engl J Med 377:162-172. https://doi.org/10.1056/NEJMra1603471

7. Butler A, Hoffman P, Smibert P, Papalexi E, Satija R (2018) Integrating single-cell transcriptomic data across different conditions, technologies, and species. Nat Biotechnol 36:411-420. https://doi.org/10.1038/nbt. 4096

8. Chen H, Richard M, Sandler DP, Umbach DM, Kamel F (2007) Head injury and amyotrophic lateral sclerosis. Am J Epidemiol 166:810-816. https:// doi.org/10.1093/aje/kwm153

9. Consortium GT, Laboratory DA, Coordinating Center -Analysis Working G, Statistical Methods groups-Analysis Working G, Enhancing Gg et al (2017) Genetic effects on gene expression across human tissues. Nature 550:204-213. https://doi.org/10.1038/nature24277

10. Cooper-Knock J, Hewitt C, Highley JR, Brockington A, Milano A, Man S, Martindale J, Hartley J, Walsh T, Gelsthorpe C et al (2012) Clinicopathological features in amyotrophic lateral sclerosis with expansions in C9ORF72. Brain 135:751-764. https://doi.org/10.1093/brain/awr365

11. Turner SD (2018) qqman: an R package for visualizing GWAS results using Q-Q and manhattan plots. J Open Source Softw. https://doi.org/10. 21105/joss.00731

12. Das S, Forer L, Schonherr S, Sidore C, Locke AE, Kwong A, Vrieze SI, Chew EY, Levy S, McGue M et al (2016) Next-generation genotype imputation service and methods. Nat Genet 48:1284-1287. https://doi.org/10.1038/ ng.3656

13. Gagliano SA, Ptak C, Mak DYF, Shamsi M, Oh G, Knight J, Boutros PC, Petronis A (2016) Allele-Skewed DNA Modification in the Brain: Relevance to a Schizophrenia GWAS. Am J Hum Genet 98:956-962. https://doi.org/ 10.1016/j.ajhg.2016.03.006

14. Gijselinck I, Van Mossevelde S, van der Zee J, Sieben A, Engelborghs S, De Bleecker J, Ivanoiu A, Deryck O, Edbauer D, Zhang M et al (2016) The C9orf72 repeat size correlates with onset age of disease, DNA methylation and transcriptional downregulation of the promoter. Mol Psychiatry 21:1112-1124. https://doi.org/10.1038/mp.2015.159

15. Hannon E, Spiers H, Viana J, Pidsley R, Burrage J, Murphy TM, Troakes C, Turecki G, O'Donovan MC, Schalkwyk LC et al (2016) Methylation QTLs in the developing brain and their enrichment in schizophrenia risk loci. Nat Neurosci 19:48-54. https://doi.org/10.1038/nn.4182

16. Hardy J, Rogaeva E (2014) Motor neuron disease and frontotemporal dementia sometimes related, sometimes not. Exp Neurol 262:75-83

17. Hemonnot AL, Hua J, Ulmann L, Hirbec H (2019) Microglia in alzheimer disease: well-known targets and new opportunities. Front Aging Neurosci 11:233. https://doi.org/10.3389/fnagi.2019.00233

18. Kunkle BW, Grenier-Boley B, Sims R, Bis JC, Damotte V, Naj AC, Boland A, Vronskaya M, van der Lee SJ, Amlie-Wolf A et al (2019) Genetic meta-analysis of diagnosed Alzheimer's disease identifies new risk loci and implicates Abeta, tau, immunity and lipid processing. Nat Genet 51:414-430. https://doi.org/10.1038/s41588-019-0358-2

19. Lek M, Karczewski KJ, Minikel EV, Samocha KE, Banks E, Fennell T, O'Donnell-Luria AH, Ware JS, Hill AJ, Cummings BB et al (2016) Analysis of protein-coding genetic variation in 60,706 humans. Nature 536:285-291. https://doi.org/10.1038/nature19057

20. Lim U, Song MA (2012) Dietary and lifestyle factors of DNA methylation. Methods Mol Biol 863:359-376. https://doi.org/10.1007/978-1-61779612-8_23

21. Mackenzie IR, Arzberger T, Kremmer E, Troost D, Lorenzl S, Mori K, Weng SM, Haass C, Kretzschmar HA, Edbauer D et al (2013) Dipeptide repeat protein pathology in C9ORF72 mutation cases: clinico-pathological 
correlations. Acta Neuropathol 126:859-879. https://doi.org/10.1007/ s00401-013-1181-y

22. McCarthy S, Das S, Kretzschmar W, Delaneau O, Wood AR, Teumer A, Kang HM, Fuchsberger C, Danecek P, Sharp K et al (2016) A reference panel of 64,976 haplotypes for genotype imputation. Nat Genet 48:1279-1283. https://doi.org/10.1038/ng.3643

23. Nicolas A, Kenna KP, Renton AE, Ticozzi N, Faghri F, Chia R, Dominov JA, Kenna BJ, Nalls MA, Keagle P et al (2018) Genome-wide Analyses Identify KIF5A as a Novel ALS Gene. Neuron 97(1268-1283):e1266. https://doi. org/10.1016/j.neuron.2018.02.027

24. Pislar A, Kos J (2014) Cysteine cathepsins in neurological disorders. Mol Neurobiol 49:1017-1030. https://doi.org/10.1007/s12035-013-8576-6

25. Renton AE, Majounie E, Waite A, Simon-Sanchez J, Rollinson S, Gibbs JR, Schymick JC, Laaksovirta H, van Swieten JC, Myllykangas L et al (2011) A hexanucleotide repeat expansion in C9ORF72 is the cause of chromosome 9p21-linked ALS-FTD. Neuron 72:257-268. https://doi.org/10. 1016/j.neuron.2011.09.010

26. Riese RJ, Mitchell RN, Villadangos JA, Shi GP, Palmer JT, Karp ER, De Sanctis GT, Ploegh HL, Chapman HA (1998) Cathepsin S activity regulates antigen presentation and immunity. J Clin Invest 101:2351-2363. https://doi.org/ 10.1172/JCl1158

27. Russ J, Liu EY, Wu K, Neal D, Suh E, Irwin DJ, McMillan CT, Harms MB, Cairns NJ, Wood EM et al (2015) Hypermethylation of repeat expanded C9orf72 is a clinical and molecular disease modifier. Acta Neuropathol 129:39-52. https://doi.org/10.1007/s00401-014-1365-0

28. Schmahmann JD, Guell X, Stoodley CJ, Halko MA (2019) The theory and neuroscience of cerebellar cognition. Annu Rev Neurosci 42:337-364. https://doi.org/10.1146/annurev-neuro-070918-050258

29. Sousa C, Golebiewska A, Poovathingal SK, Kaoma T, Pires-Afonso Y, Martina S, Coowar D, Azuaje F, Skupin A, Balling R et al (2018) Single-cell transcriptomics reveals distinct inflammation-induced microglia signatures. EMBO Rep. https://doi.org/10.15252/embr.201846171

30. Swinnen B, Robberecht W (2014) The phenotypic variability of amyotrophic lateral sclerosis. Nat Rev Neurol 10:661-670. https://doi.org/10. 1038/nrneurol.2014.184
31. Wang H, O'Reilly EJ, Weisskopf MG, Logroscino G, McCullough ML, Thun MJ, Schatzkin A, Kolonel LN, Ascherio A (2011) Smoking and risk of amyotrophic lateral sclerosis: a pooled analysis of 5 prospective cohorts. Arch Neurol 68:207-213. https://doi.org/10.1001/archneurol.2010.367

32. Xi Z, Zinman L, Moreno D, Schymick J, Liang Y, Sato C, Zheng Y, Ghani M, Dib S, Keith J et al (2013) Hypermethylation of the CpG island near the G4C2 repeat in ALS with a C9orf72 expansion. Am J Hum Genet 92:981-989. https://doi.org/10.1016/j.jhg.2013.04.017

33. Zhang M, Dilliott AA, Khallaf R, Robinson JF, Hegele RA, Comishen M, Sato C, Tosto G, Reitz C, Mayeux R et al (2019) Genetic and epigenetic study of an Alzheimer's disease family with monozygotic triplets. Brain J Neurol 142:3375-3381. https://doi.org/10.1093/brain/awz289

34. Zhang M, Ferrari R, Tartaglia MC, Keith J, Surace El, Wolf U, Sato C, Grinberg M, Liang Y, Xi Z et al (2018) A C6orf10/LOC101929163 locus is associated with age of onset in C9orf72 carriers. Brain 141:2895-2907. https:// doi.org/10.1093/brain/awy238

35. Zhang M, McKeever PM, Xi Z, Moreno D, Sato C, Bergsma T, McGoldrick P, Keith J, Robertson J, Zinman L et al (2020) DNA methylation age acceleration is associated with ALS age of onset and survival. Acta Neuropathol 139:943-946. https://doi.org/10.1007/s00401-020-02131-z

36. Zhang M, Tartaglia MC, Moreno D, Sato C, McKeever P, Weichert A, Keith J, Robertson J, Zinman L, Rogaeva E (2017) DNA methylation age-acceleration is associated with disease duration and age at onset in C9orf72 patients. Acta Neuropathol 134:271-279. https://doi.org/10.1007/ s00401-017-1713-y

37. Zhang M, Xi Z, Ghani M, Jia P, Pal M, Werynska K, Moreno D, Sato C, Liang Y, Robertson J et al (2016) Genetic and epigenetic study of ALS-discordant identical twins with double mutations in SOD1 and ARHGEF28. J Neurol Neurosurg Psychiatry 87:1268-1270. https://doi.org/10.1136/ jnnp-2016-313592

\section{Publisher's Note}

Springer Nature remains neutral with regard to jurisdictional claims in published maps and institutional affiliations.
Ready to submit your research? Choose BMC and benefit from:

- fast, convenient online submission

- thorough peer review by experienced researchers in your field

- rapid publication on acceptance

- support for research data, including large and complex data types

- gold Open Access which fosters wider collaboration and increased citations

- maximum visibility for your research: over $100 \mathrm{M}$ website views per year

At BMC, research is always in progress.

Learn more biomedcentral.com/submissions 